\title{
HONGOS MICORRÍZICOS ARBUSCULARES Y FERTILIZACIÓN EN Heliconia psittacorum L. F. $\times$ H. spathocircinata CV. TROPICS
}

\author{
ARBUSCULAR MYCORRHIZAL FUNGI AND FERTILIZATION IN Heliconia \\ psittacorum L. F. $\times$ H. spathocircinata CV. TROPICS
}

\author{
Obdulia Baltazar-Bernal* y David Jaén-Contreras
}

Colegio de Postgraduados, Campus Córdoba, Amatlán de los Reyes, Veracruz, México.

*Autor de correspondencia (obduliabb@colpos.mx)

\section{RESUMEN}

La Heliconia L.f psittacorum $\times \mathrm{H}$. spathocircinata es una planta de uso ornamental muy demandada a nivel nacional e internacional. Uno de los factores más importantes en el crecimiento vegetativo de plantas de heliconia es el aporte de macronutrientes, los cuales influyen de manera importante en el rendimiento y calidad de flores. Se estableció el cv. Tropics de heliconia a cielo abierto en un arreglo de $1 \mathrm{~m}$ entre surcos y $1.5 \mathrm{~m}$ entre plantas para evaluar el efecto de la inoculación, por separado, de 350 esporas de Entrophospora colombiana y Funneliformis mosseae por planta sobre el crecimiento vegetativo del cultivo. Al momento de la plantación se fertilizó con las dosis de 100-0-150, 100-30-150 y 100-60-150 kg ha-1 de N-P-K. Los resultados mostraron que E. colombiana + 100-30-150 N-P-K estimularon el crecimiento vegetativo de Heliconia cv. Tropics; se incrementó la altura de la planta, diámetro del tallo, número de brotes, área foliar, contenido de clorofila y peso seco radical con respecto al testigo. La dosis de 100-0-150 N-P-K incrementó significativamente el peso seco aéreo, el peso seco de la raíz y la materia seca total. Los resultados mostraron que E. colombiana no fue tan eficaz en la colonización micorrízica del sistema radical de las plantas de heliconia en comparación con $F$. mosseae, que indujo los mayores porcentajes de colonización. Se encontró que $E$. colombiana produjo significativamente más esporas (1240) en comparación con $F$. mosseae (753); también se observó que a mayor concentración de $\mathrm{P}$ en la fertilización el número de esporas de E. colombiana disminuyó proporcionalmente, mientras que con F. mosseae la respuesta fue diferente. Se concluye que la inoculación con E. colombiana junto con una dosis de fertilización de 100-30-150 NPK tiene mayor potencial para incrementar la producción de Heliconia cv. Tropics.

Palabras clave: Entrophospora colombiana, Funneliformis mosseae, crecimiento vegetativo. heliconia.

\section{SUMMARY}

Heliconia psittacorum L. f. $\times \mathrm{H}$. spathocircinata is an ornamental plant that is highly demanded nationally and internationally. One of the most important factors in the vegetative growth of heliconia plants is the provision of macronutrients, which have an important influence on yield and quality of flowers. Heliconia cv. Tropics was established in an open field plantation under an arrangement of $1 \mathrm{~m}$ between rows and $1.5 \mathrm{~m}$ between plants to separately evaluate the effect of the inoculation of 350 spores of Entrophospora colombiana and Funneliformis mosseae per plant on the vegetative growth of the plants. At the time of planting fertilization was applied in doses of 100-0-150, 100-30-150 and 100-60-150 kg ha-1 of N-P-K. Results showed that E. colombiana $+100-30-150 \mathrm{~N}-\mathrm{P}-\mathrm{K}$ stimulated the vegetative growth of Heliconia cv. Tropics; plant height, stem diameter, number of shoots, leaf area, chlorophyll content and root dry weight increased compared to the control. The dose 100-0-150 N-P-K significantly increased aerial dry weight, root dry weight and total dry matter. Results showed that E. colombiana was not as effective in the mycorrhizal colonization of the root system of heliconia plants compared to $F$. mosseae, which induced the highest rates. It was found that E. colombiana produced significantly more spores (1240) compared to F. mosseae (753); it was also observed that at higher concentration of $P$ in fertilization the number of spores of $E$. colombiana decreased proportionally, while with $F$. mosseae the response was different. It is concluded that the inoculation with E. colombiana along with a fertilization dose of 100-30-150 NPK has greater potential to increase the production of Heliconia cv. Tropics.

Index words: Entrophospora colombiana, Funneliformis mosseae, heliconia, vegetative growth

\section{INTRODUCCIÓN}

Heliconia L.f psittacorum $\times$ H. spathocircinata cv. Tropics (Heliconia cv. Tropics) es una planta herbácea tropical perenne que se destaca por su producción durante todo el año y por su bella y duradera inflorescencia (Baltazar et al., 2011), atributos que la han convertido en un producto ornamental muy demandado a nivel nacional e internacional. El aporte de macronutrientes es uno de los factores más importantes en el crecimiento vegetativo de plantas de heliconia, que influyen de manera importante en el rendimiento y calidad de flores. La escasez de un nutriente puede causar anomalías visibles y afectar la producción de flores (Malavolta, 2006). La heliconia es un cultivo que generalmente requiere altas tasas de macroelementos, particularmente $\mathrm{N}$; sin embargo, a nivel comercial existe una gran variación en los sistemas de fertilización (De Castro et al., 2015). El intenso crecimiento de heliconia y su alta producción de tallos vegetativos y de área foliar requiere de la aplicación de $200 \mathrm{~g} \mathrm{~m}^{-2}$ de N-P-K (Nitrofoska ${ }^{\circledR} 22+8+10$ ) cada tres meses (De Farías et al., 2013). En otro estudio se reportó la aplicación de 0 , 120, 180 y $240 \mathrm{~g}$ de $\mathrm{N}$ y K, y de $45 \mathrm{~g}$ de P por punto de plantación, aplicaciones que fueron divididas a los tres, seis y nueve meses después de la plantación (Amaral et al., 2015). En dalia (Dahlia $\times$ hibrida) también se ha cotejado 
que los protocolos de producción incluyen el uso de altas cantidades de fertilizantes solubles en los sustratos para lograr un suministro óptimo de nutrientes como $\mathrm{N}$ y $\mathrm{P}$ en un corto periodo de crecimiento, además de pesticidas en plena floración, lo que origina un impacto en los costos de producción (Younis et al., 2006). Para superar este problema, además de la fertilización inorgánica o convencional, como alternativa para el aporte de macro y micronutrientes, se tiene el uso de abonos orgánicos de origen animal o vegetal, así como de vermicomposta, biofertilizantes y el empleo de microorganismos (AguilarGonzález et al., 2015).

Diversos estudios en otras especies han demostrado la eficacia de los hongos micorrízicos arbusculares (HMA) en el crecimiento vegetativo de las plantas ornamentales y no ornamentales (Montoya-Martínez et al., 2019), incrementando el crecimiento y desarrollo de las mismas en términos de altura (Long et al., 2010; Sohn et al., 2003), diámetro de tallo (El-Khateeb et al., 2011) y número de flores (Perner et al., 2007; Scagel, 2003; Scagel y Schreiner, 2006). Los HMA cumplen la función de mejorar la absorción de los nutrientes del suelo principalmente P, Cu y Zn (Cardoso y Kuyper, 2006; Prasad et al., 2012); así, algunos estudios con HMA han reportado que promueven el crecimiento y el desarrollo de plantas como Acacia saligna (El-Khateeb et al., 2011), Eustoma grandiflorum (Meir et al., 2010) y Lilium sp. (Rubí et al., 2009); asimismo, Vaingankar y Rodrigues (2015) reportaron que la inoculación con HMA en Crossandra infundibuliformis aumentó la altura de la planta, el número de flores, la concentración de P en las hojas y el peso seco total de la planta; además, indujo la floración temprana. Gaur et al. (2000) reportaron que se incrementó la concentración de $\mathrm{P}$ y $\mathrm{K}$ en los brotes con la inoculación de hongos micorrízicos en Petunia hybrida, Callistephus chinensis e Impatiens balsamina y se incrementó el número de flores y mejoró el desarrollo vegetativo de las plantas (Poulton et al., 2002).

Las investigaciones de Tawaraya et al. (2012) y de Zhang et al. (2009) consolidan los argumentos de que la aplicación de los HMA tiene el potencial de reducir el uso de fertilizantes químicos en gran escala. Por lo anterior, el efecto benéfico de los HMA en sistemas de producción intensivos de plantas ornamentales es dependiente de factores como la fertilización. El objetivo del presente estudio fue evaluar el efecto por separado de la inoculación de 350 esporas de Entrophospora colombiana y Funneliformis mosseae, así como la aplicación de tres dosis de fertilización de N-P-K sobre el crecimiento vegetativo de plantas de heliconia.

\section{MATERIALES Y MÉTODOS}

\section{Área de estudio}

La etapa de campo se desarrolló en una plantación de heliconias en el Colegio de Postgraduados, Campus Córdoba, ubicado a $18^{\circ} 46^{\prime} \mathrm{N}$ y $96^{\circ} 55^{\prime} \mathrm{O}$, a 720 msnm, de agosto a diciembre de 2016. El clima del lugar es cálido húmedo, con abundantes lluvias en verano y temperatura media de 22 y máxima de $35^{\circ} \mathrm{C}$; la precipitación promedio es de 1,807 mm anuales; el suelo es un vertisol con una elevada proporción de arcillas esmectíticas (INEGI, 2015), con $\mathrm{pH}$ de 5.7.

\section{Material vegetal}

Se utilizaron plantas de heliconia cultivar Tropics obtenidas de plantas madre con cuatro a cinco hijuelos de $1.20 \mathrm{~m}$ de altura. Las plantas fueron cosechadas con base en su vigor, con tres brotes y se cortaron a $30 \mathrm{~cm}$ de altura; posteriormente, se les eliminaron las hojas y los hijuelos antes de plantar y se les aplicó un riego pesado.

\section{Manejo agronómico}

Se realizó barbecho, rastreo y surcado con distancia de $1 \mathrm{~m}$ entre surcos el 20 de julio de 2016. El suelo se desinfectó con el fumigante $\mathrm{N}$-Metil ditiocarbamato de sodio (Bunema ${ }^{\circledR} 55$ GE, Buckman, México) a dosis de $1 \mathrm{~L} \mathrm{ha}^{-1}$ disuelto en $185 \mathrm{~L}$ de agua; este producto tiene acción inhibitoria contra Fusarium spp. y Phytophthora spp. que causan pudrición radical y pudrición de cuello y tallo, respectivamente (Cremlyn, 1991). Posteriormente se colocó un acolchado con plástico calibre 300 de color blanco en la parte superior y negro en la inferior y la plantación se hizo a $1.5 \mathrm{~m}$ entre plantas.

\section{Aislamiento de las esporas}

Las esporas de los HMA Funneliformis mosseae y Entrophospora colombiana fueron aisladas de la rizósfera de un suelo de un huerto de mango (Mangifera indica L.) en el municipio de Manlio Fabio Altamirano, Veracruz, México. Las esporas se extrajeron mediante el método de tamizado húmedo (Números de Tamiz: 44, 325 y 400 m) y decantación (Gerdemann y Nicolson 1963). Se realizó una agrupación morfológica considerando forma, color y tamaño (INVAM, 2016). Los morfotipos obtenidos se inocularon en plántulas de trigo (Triticum aestivum L.) (una espora por planta, 10 repeticiones). El cultivo monospórico se estableció en macetas de $1 \mathrm{~L}$ de capacidad y como sustrato arena estéril. Las plantas se mantuvieron en 
invernadero a una temperatura promedio de $40^{\circ} \mathrm{C}$. Se aplicó solución nutritiva (Steiner, 1973) modificada en fósforo $20 \%$, pH 7.5, aplicando riegos diariamente. Se realizaron muestreos de suelo mensualmente para evaluar la propagación de la espora inoculada de los diferentes morfotipos; en el cuarto mes se colocó una semilla de trigo por maceta para un segundo ciclo de cultivo con la finalidad de que la espora se multiplicara y estableciera simbiosis. En los dos últimos meses las plantas se sometieron a estrés hídrico suspendiendo totalmente el riego con el objeto de acelerar dicha simbiosis; posteriormente, las esporas de Ios HMA se extrajeron de las muestras de arena mediante el método de Gerdemann y Nicolson (1963).

La identificación de F. mosseae y E. colombiana se hizo con base en la descripción de los grupos de paredes de las esporas, en preparaciones semipermanentes utilizando alcohol polivínilico, ácido láctico, glicerol con y sin reactivo de Melzer (Schenck y Pérez, 1990). En la producción del inoculante de los HMA mencionados, las esporas fueron desinfectadas en la superficie de la pared con una solución de Cloramina $\mathrm{T}\left(20 \mathrm{~g} \mathrm{~L}^{-1}\right)$ con estreptomicina (200 $\left.\mathrm{mg} \mathrm{L}^{-1}\right)$; posteriormente, y de acuerdo con el método de plantas hospedantes (Sieverding, 1983), fueron inoculadas a plantas de sorgo (Sorghum vulgare L.) desarrolladas en arena de río estéril $\left(120^{\circ} \mathrm{C}, 3\right.$ horas por tres días consecutivos). Después de cuatro meses se realizó la cosecha, se determinó la tinción de raíz con el método de Phillips y Hayman (1970), y con el método de intersección de cuadrantes de Giovannetti y Mosse (1980) se midió el porcentaje de colonización micorrízica en la raíz, que fue de $87.5 \%$ para F. mosseae y de $97.5 \%$ para E. colombiana.

\section{Inoculación de esporas}

La inoculación con los micorrízicos se hizo al momento de la plantación, agregando 350 esporas de F. mosseae y 350 esporas de E. colombiana respectivamente, por planta, contenidas en $10 \mathrm{~g}$ de sustrato inóculo. La cantidad de esporas mencionadas se agregó debido a que se desconocía la infectividad y capacidad de cada hongo micorrízico para penetrar e invadir la raíz de la heliconia y explorar el suelo, así como su habilidad de persistir en ese sistema productivo. Las esporas se colocaron sobre el sistema radical de cada una de las plantas para garantizar el contacto con la raíz.

\section{Diseño y unidad experimental}

\section{Diseño experimental}

Se utilizó un diseño experimental completamente al azar, con 10 tratamientos y 10 repeticiones; la unidad experimental fue de una planta por hoyo. Los tratamientos utilizados, así como su descripción, se encuentran en el Cuadro 1. Posterior a la inoculación, a los tratamientos se les aplicó la correspondiente dosis de N-P-K en cada hoyo de plantación.

\section{Variables evaluadas}

A los 150 días después de la plantación (DDP) se determinaron las variables 1) altura de la planta en $\mathrm{cm}$, de la base del tallo al botón apical; 2) diámetro del tallo en $\mathrm{mm}$, a $10 \mathrm{~cm}$ de la base con un vernier digital Caldi-6MO

Cuadro 1. Hongos micorrízicos y fertilizantes aplicados a Heliconia cv. Tropics.

\begin{tabular}{|c|c|c|c|c|c|}
\hline \multirow[b]{2}{*}{ Tratamiento } & \multirow[b]{2}{*}{ Micorriza $^{+}$} & \multirow{2}{*}{$\begin{array}{c}\text { Dosis de } \\
\mathrm{N}-\mathrm{P}_{2} \mathrm{O}_{5}-\mathrm{K}_{2} \mathrm{O}\end{array}$} & \multicolumn{3}{|c|}{ Fertilizantes (g) } \\
\hline & & & $\begin{array}{c}\text { Desarrollo® } \\
13-0-17\end{array}$ & $\begin{array}{c}\text { Nitrimon }{ }^{\circledR} \\
0-0-60\end{array}$ & $\begin{array}{c}\text { Basacote Plus } \AA \\
16-8-12\end{array}$ \\
\hline T1 & Testigo & NA & NA & NA & NA \\
\hline T2 & E. colombiana & NA & NA & NA & NA \\
\hline T3 & E. colombiana & 100-30-150 & 41.6 & 12.0 & 5.0 \\
\hline T4 & E. colombiana & $100-60-150$ & NA & 13.5 & 10 \\
\hline T5 & NA & 100-0-150 & 103.8 & 4.3 & NA \\
\hline T6 & NA & $100-30-150$ & 41.6 & 12.0 & 5.0 \\
\hline T7 & NA & $100-60-150$ & NA & 13.5 & 10 \\
\hline T8 & F. mosseae & NA & NA & NA & NA \\
\hline T9 & F. mosseae & 100-30-150 & 41.6 & 12.0 & 5.0 \\
\hline T10 & F. mosseae & $100-60-150$ & NA & 13.5 & 10 \\
\hline
\end{tabular}

${ }^{\dagger}$ Dosis de micorriza: 350 esporas por especie, NA: no se aplicó micorriza o fertilizante. 
(Truper, México); 3) número de brotes por planta; 4) área foliar en $\mathrm{cm}^{2}$, se midió introduciendo las hojas en un integrador de área foliar (Licor Modelo 3100 Area Meter ${ }^{\circledR}$, Lincoln, Nebraska, EUA); 5) clorofila por unidad de área foliar en $\mathrm{mg} \mathrm{cm}^{-2}$, en un medidor de clorofila (Spad CCM200 plus ${ }^{\circledR}$ Modelo FE-242A, Opti-Sciences, Hudson, New Hampshire, EUA); 6) los pesos secos de la raíz, el tallo y las hojas se obtuvieron en $\mathrm{g}$, poniendo a secar las estructuras a $70{ }^{\circ} \mathrm{C}$ en una estufa Modelo No. 3471-1 (Thermo Scientific, Waltham, Massachusetts, EUA) hasta peso constante; 7) el porcentaje de colonización micorrízica se determinó en las raíces de las plantas de cada uno de los tratamientos de acuerdo con el método descrito por Phillips y Hayman (1970) para tinción de raíces y el método de intersección de cuadrantes (Giovannetti y Mosse, 1980), que permite calcular el porcentaje de colonización micorrízica considerando la proporción de intersecciones micorrizadas con respecto al total de intersecciones registradas utilizando un microscopio de disección (45 X); 8) la evaluación del número de esporas se realizó separando las esporas con tamices de 44, 325 y $400 \mu \mathrm{m}$ por el método de Gerdemann y Nicolson (1963) utilizando un microscopio estereoscópico (7 a 60 X).

\section{Análisis estadístico}

Los datos se analizaron mediante análisis de varianza y la separación de medias fue realizada con la diferencia significativa honesta $(D S H)$ de Tukey $(P \leq 0.05)$ utilizando el programa Statistical Analysis System, versión 9.3 (SAS Institute, 2011).

\section{RESULTADOS Y DISCUSIÓN}

En el presente estudio se demostró que la inoculación individual de los hongos endomicorrízicos E. colombiana y F. mosseae causaron efectos diferentes en la fisiología de las plantas de heliconia, manifestado en el crecimiento de las plantas; el hongo que indujo mayor eficiencia en la fisiología de las plantas fue E. colombiana, igualando en el efecto sobre la altura, diámetro de tallo, número de brotes y contenido de clorofila a las tres dosis de fertilización aplicadas (100-0-150, 100-30-150 y 100-60-150 N-P-K) y superando a todos los tratamientos en área foliar (Cuadro 2). Estos resultados permiten afirmar que para lograr plantas de calidad en heliconia, no sólo es necesaria la aplicación de fertilizantes sintéticos (Sosa, 2013), sino que la incorporación de los hongos micorrízicos en la tecnología de viveros de plantas ornamentales es muy deseable por los beneficios directos observados en el crecimiento vegetativo y reproductivo de las plantas (Gaur y Adholeya, 2005), ya que inducen crecimientos similares a los ocasionados por las dosis de fertilización química, por lo que se reducen significativamente los costos de producción (Alarcón y Ferrera-Cerrato, 1999) (Cuadro 2). Aparentemente, las especies de hongos micorrízicos no tienen especificidad en la elección de su hospedero (Smith y Read, 2008); sin embargo, las diferencias en los efectos que las dos especies de hongos micorrízicos causaron sobre el crecimiento de las plantas de heliconia indican que éstas responden específicamente a algunas especies de hongos micorrízicos (Van der Heijden et al., 1998). En las condiciones edafoclimáticas bajo las que se desarrolló la presente investigación, E. colombiana benefició en mayor grado el crecimiento de las plantas de heliconia. Sieverding y Toro (1985) mostraron que este hongo es muy eficiente en la colonización micorrízica y en el crecimiento y desarrollo de plantas en suelos que presentan alto nivel de acidez y se adapta a diversos niveles de fertilidad; por el contrario, Glomus mosseae (hoy Funneliformis mosseae) no es tolerante a pH por debajo de 5 (Mosse, 1972; 1973). Lo anterior permite afirmar que los máximos beneficios de la inoculación micorrízica se obtienen utilizando los hongos micorrízicos arbusculares más eficaces tras una cuidadosa selección de combinaciones planta-hongos micorrízicos-sustrato altamente compatibles (Sosa et al., 2006).

En el presente estudio se observó que los hongos micorrízicos tienden a disminuir la necesidad de fertilizante químico por contribuir a la demanda de los niveles de NPK óptimos para el crecimiento de las plantas. Al respecto, existen evidencias de que el micelio de los hongos micorrízicos tiene la capacidad para absorber y transferir nutrimentos como N, K, Ca, Mg, Si, Cu, Zn, B y Fe, dado que se encuentran concentraciones más altas de estos elementos en las plantas micorrizadas (Marschner y Dell, 1994; Nakano et al., 2001); no obstante, también se debe considerar que los iones de fosfato inorgánico se unen rápidamente a coloides del suelo o se fijan como sales de fierro o aluminio, volviéndose relativamente inmóviles, además de que una gran proporción del fósforo inorgánico total está normalmente en forma insoluble, no disponible fácilmente para las plantas.

Lo anterior permite explicar que la respuesta de la planta de heliconia al suministro de fertilizante al suelo se encubrió por la baja solubilidad de los nutrimentos bajo estas condiciones. En otras investigaciones con diferentes plantas y hongos micorrízicos ha quedado demostrada la eficacia de estos hongos para estimular crecimientos significativos; por ejemplo, El-Khateeb et al. (2011) encontraron que Glomus sp. incrementó la altura de la planta, el diámetro del tallo y el área foliar de Acacia saligna en $88.6,88.6$ y 85.6 \% en relación con el testigo. En otro estudio, Gaur et al. (2000) demostraron que plantas de Petunia hybrida, Impatiens balsamina y Callistephus chinensis inoculadas con el consorcio de 
Glomus, Gigaspora y Scutellospora spp. obtuvieron 65.9 $\%$ de altura en comparación con las plantas testigo, que únicamente lograron $56.4 \%$. Asrar et al. (2012) utilizaron Glomus deserticola en plantas de Antirhinum majus L. obteniendo $89 \%$ de la altura, $74 \%$ del diámetro y $82 \%$ del área foliar con respecto al testigo. Los resultados del presente estudio también demostraron que la interacción de E. colombiana con la dosis de fertilización 100-30-150 NPK resultó más efectiva que las dosis de 100-0-150, 10030-150 y 100-60-150 para incrementar la altura de las plantas, el diámetro del tallo, el número de brotes, el área foliar y el contenido de clorofila en heliconia (Cuadro 2). Estos resultados sugieren que la combinación racional de los hongos micorrízicos, específicamente E. colombiana y la dosis de fertilización 100-30-150 de NPK puede ser ventajosa para la producción comercial de plantas de heliconia. Estudios previos (Poulton et al., 2002; Tawaraya et al., 2012; Zhang et al., 2009) consolidan la idea de que la aplicación de hongos micorrízicos tiene el potencial para reducir el uso de fertilizantes químicos en gran escala y representa una posibilidad real de mejorar la economía del productor de plantas de heliconias.

Adicionalmente, los resultados mostraron que la dosis de fertilización 100-0-150 fue muy eficiente para incrementar significativamente el peso seco aéreo, el peso seco de la raíz y la materia seca total (Cuadro 3). Este efecto favorable puede ser explicado considerando que el nitrógeno es uno de los elementos más esenciales para las plantas y origina la producción de proteínas adicionales y permite que las hojas de las plantas crezcan más y tengan mayor superficie disponible para la fotosíntesis; adicionalmente, el nitrógeno también influye en la proliferación de la biomasa de la raíz, resultando en una mayor absorción de nutrientes y de agua del suelo, lo que conduce a una mayor biomasa (Arisha y Bardisi, 1999); contrariamente, el potasio es insignificante en el peso seco total de la planta. Resultados similares fueron encontrados por Sahar et al. (2009), quienes indicaron que la fertilización con potasio incrementó el crecimiento de la planta y el rendimiento de Thuja orientalis. El no haber encontrado un efecto positivo de la inoculación de los hongos micorrízicos en la biomasa de las plantas de heliconia no es negativo, ya que las plantas necesitan destinar una cantidad suficiente de asimilados para los demás órganos, con el fin de mantener su capacidad de producción (Kuiper, 1993).

Finalmente, cabe resaltar las diferencias de los dos hongos micorrízicos en la colonización radical y en el número de esporas, encontrándose que F. mosseae fue más infectivo en colonizar el sistema radical de las plantas de heliconia y también resultó ser más sensible a las dosis de fertilización que E. colombiana; sin embargo, este último hongo fue más eficiente y efectivo para inducir una mejor respuesta en la mayoría de las variables evaluadas (Cuadro 4). Al respecto, Varma y Schüepp (1994) mencionan que los hongos micorrízicos pueden colonizar intensamente las raíces sin una promoción proporcional del crecimiento de las plantas, lo que puede resultar en una pérdida de fotosintatos sin una mejora compensatoria en la nutrición mineral de la planta hospedera (Clapperton y Reid, 1992). Abbott et al. (1992) mencionaron que la efectividad de los hongos micorrízicos no siempre está correlacionada con la capacidad infectiva de éstos.

Cuadro 2. Efecto de la inoculación con E. colombiana y F. mosseae y la aplicación de N-P-K en el crecimiento de Heliconia cv. Tropics, a los 125 días después de la inoculación.

\begin{tabular}{|c|c|c|c|c|c|c|}
\hline Micorriza $^{+}+\mathrm{N}-\mathrm{P}-\mathrm{K}$ & $\begin{array}{c}\text { Altura de } \\
\text { planta }(\mathrm{cm})\end{array}$ & $\begin{array}{c}\text { Diámetro del } \\
\text { tallo }(\mathrm{mm})\end{array}$ & $\begin{array}{c}\text { Número de } \\
\text { brotes }\end{array}$ & $\begin{array}{l}\text { Área } \\
\text { (cr }\end{array}$ & liar & $\begin{array}{c}\text { Clorofila } \\
\left(\mathrm{mg} \mathrm{cm}^{-2}\right)\end{array}$ \\
\hline Testigo & $49.37 \mathrm{bc}$ & $24.64 a b$ & $3.40 \mathrm{ab}$ & 560.20 & bc & $26.55 \mathrm{ab}$ \\
\hline E. colombiana & $57.50 \mathrm{abc}$ & 29.31 a & $4.90 a b$ & 735.40 & $a b$ & 30.41 a \\
\hline E. colombiana + 100-30-150 & 68.45 a & $31.60 \mathrm{a}$ & 6.60 a & 1072.6 & a & 27.9 a \\
\hline E. colombiana + 100-60-150 & $56.45 \mathrm{abc}$ & $24.63 \mathrm{ab}$ & $3.1 \mathrm{~b}$ & 602.2 & bc & $20.75 a b$ \\
\hline 100-0-150 & $48.13 \mathrm{bc}$ & $18.45 \mathrm{~b}$ & $4.60 \mathrm{ab}$ & 473.20 & bc & $19.13 \mathrm{ab}$ \\
\hline 100-30-150 & $58.52 a b$ & $26.73 a b$ & $4.60 \mathrm{ab}$ & 545.90 & bc & $18.21 \mathrm{ab}$ \\
\hline 100-60-150 & $58.45 a b$ & $28.11 \mathrm{ab}$ & $3.60 \mathrm{ab}$ & 516.00 & bc & $18.96 \mathrm{ab}$ \\
\hline F. mosseae & $49.40 \mathrm{bc}$ & $24.85 a b$ & $3.70 a b$ & 435.40 & bc & $22.92 \mathrm{ab}$ \\
\hline F. mosseae + 100-30-150 & $45.85 \mathrm{bc}$ & $21.69 \mathrm{ab}$ & $3.1 \mathrm{~b}$ & 410.4 & bc & $15.24 \mathrm{~b}$ \\
\hline F. mosseae + 100-60-150 & $41.24 \mathrm{c}$ & $21.51 \mathrm{ab}$ & $2.7 \mathrm{~b}$ & 318.3 & c & $14.94 \mathrm{~b}$ \\
\hline
\end{tabular}

${ }^{+}$Dosis de micorriza: 350 esporas por planta. Medias con la misma letra dentro de cada columna no son estadísticamente diferentes (Tukey, $\mathrm{P} \leq 0.05$ ). 
Lo anterior confirma que F. mosseae resultó muy infectivo pero no indujo efectos significativos en el crecimiento de las plantas de heliconia; en contraste, E. colombiana resultó ser muy eficiente al estimular efectos altamente significativos en todas las variables evaluadas, pero no fue tan infectivo en la colonización radical de las plantas de heliconia.

\section{CONCLUSIONES}

La inoculación con E. colombiana tiene el potencial para incrementar el crecimiento de heliconias, ya que favoreció el crecimiento vegetativo de las plantas en comparación con F. mosseae; además, su interacción con la dosis de fertilización 100-30-150 representa la mejor combinación

Cuadro 3. Efecto de inoculación con E. colombiana y F. mosseae y la aplicación de N-P-K en el peso seco de Heliconia cv. Tropics, a los 125 días después de la inoculación.

\begin{tabular}{lrrr}
\hline Micorriza $^{\dagger}+$ N-P-K & Peso seco aéreo $(\mathrm{g})$ & Peso seco de raíz (g) & Materia seca total $(\mathrm{g})$ \\
\hline Testigo & $5.26 \mathrm{~d}$ & $4.94 \mathrm{bcd}$ & $10.20 \mathrm{~b}$ \\
E. colombiana & $9.8 \mathrm{~cd}$ & $6.71 \mathrm{abcd}$ & $16.59 \mathrm{~b}$ \\
E. colombiana +100-30-150 & $18.58 \mathrm{ab}$ & $11.01 \mathrm{a}$ & $29.59 \mathrm{a}$ \\
E. colombiana +100-60-150 & $12.58 \mathrm{bc}$ & $7.49 \mathrm{abcd}$ & $20.07 \mathrm{ab}$ \\
100-0-150 & $20.90 \mathrm{a}$ & $10.10 \mathrm{ab}$ & $31.00 \mathrm{a}$ \\
100-30-150 & $7.54 \mathrm{~cd}$ & $6.53 \mathrm{abcd}$ & $14.07 \mathrm{~b}$ \\
100-60-150 & $5.41 \mathrm{~d}$ & $3.76 \mathrm{~d}$ & $9.18 \mathrm{~b}$ \\
F. mosseae & $6.31 \mathrm{~cd}$ & $3.22 \mathrm{~d}$ & $9.53 \mathrm{~b}$ \\
F. mosseae $+100-30-150$ & $7.15 \mathrm{~cd}$ & $9.42 \mathrm{abc}$ & $16.57 \mathrm{~b}$ \\
F. mosseae $+100-60-150$ & $5.98 \mathrm{~d}$ & $4.53 \mathrm{~cd}$ & $10.51 \mathrm{~b}$ \\
\hline
\end{tabular}

${ }^{\top}$ Dosis de micorriza: 350 esporas por planta. Medias con la misma letra dentro de cada columna no son estadísticamente diferentes (Tukey, $\left.\mathrm{P} \leq 0.05\right)$.

Cuadro 4. Porcentaje de colonización micorrízica, vesículas, arbúsculos, hifas y número de esporas con $E$. colombiana y $F$. mosseae y la aplicación de N-P-K en raíces de Heliconia cv. Tropics, a los 125 días después de la inoculación.

\begin{tabular}{|c|c|c|c|c|c|c|c|c|c|c|}
\hline $\begin{array}{l}\text { Micorriza }{ }^{\dagger} / \\
\text { N-P-K }\end{array}$ & CT (\%) & & V (\%) & & A (\%) & & $H(\%)$ & & $\begin{array}{l}\text { Número de } \\
\text { esporas }\end{array}$ & \\
\hline Testigo & 0.00 & c & 0.00 & $b$ & 0.00 & c & 0.00 & $b$ & 0.00 & $d$ \\
\hline E. colombiana & 70.00 & $a b$ & 11.67 & a & 39.99 & $b$ & 23.75 & a & 1240.00 & a \\
\hline $\begin{array}{l}\text { E. colombiana + } \\
100-30-150\end{array}$ & 70.00 & $a b$ & 5.83 & $a b$ & 40.00 & $b$ & 16.25 & $a b$ & 942.25 & b \\
\hline $\begin{array}{l}\text { E. colombiana + } \\
100-60-150\end{array}$ & 70.42 & $a b$ & 1.67 & $a b$ & 51.25 & $a b$ & 16.25 & $a b$ & 265.00 & $d$ \\
\hline $100-0-150$ & 0.00 & c & 0.00 & $b$ & 0.00 & c & 0.00 & $b$ & 0.00 & $d$ \\
\hline 100-30-150 & 0.00 & c & 0.00 & $b$ & 0.00 & c & 0.00 & $b$ & 0.00 & $d$ \\
\hline 100-60-150 & 0.00 & c & 0.00 & $b$ & 0.00 & c & 0.00 & $b$ & 0.00 & $d$ \\
\hline F. mosseae & 87.92 & $a b$ & 2.08 & $a b$ & 49.58 & $a b$ & 12.50 & $a b$ & 753.00 & $b c$ \\
\hline $\begin{array}{l}\text { F. mosseae + } \\
100-30-150\end{array}$ & 95.00 & a & 2.08 & $a b$ & 71.67 & a & 18.33 & a & 714.50 & bc \\
\hline $\begin{array}{l}\text { F. mosseae + } \\
100-60-150\end{array}$ & 60.41 & $b$ & 0.00 & $b$ & 32.92 & $b$ & 21.66 & a & 545.00 & c \\
\hline
\end{tabular}

${ }^{+}$Dosis de micorriza: 350 esporas por planta. Medias con la misma letra dentro de cada columna no son estadísticamente diferentes (Tukey, P $\leq$ 0.05). Ct: colonización total de micorrizas, V: vesículas, A: arbúsculos, H: hifas. 
para la producción comercial de plantas de heliconia, lo que permite ahorro de este insumo y menor contaminación por uso en exceso en los programas de fertilización química.

\section{BIBLIOGRAFÍA}

Abbott L. K., A. D. Robson and C. Gazey (1992) Selection of inoculant vesicular-arbuscular mycorrhizal fungi. Methods in Microbiology 24:1-21, https://doi.org/10.1016/S05809517(08)70084-9

Aguilar-González A. E., E. Palou and A. López-Malo (2015) Antifungal activity of essential oils of clove (Syzygium aromaticum) and/ or mustard (Brassica nigra) in vapor phase against gray mold (Botrytis cinerea) in strawberries. Innovative Food Science and Emerging Technologies 32:181-185, https://doi.org/10.1016/j. ifset 2015.09.003

Alarcón A. y R. Ferrera-Cerrato (1999) Manejo de la micorriza arbuscular en sistemas de propagación de plantas frutícolas. Terra Latinoamericana 17:179-191.

Amaral G. C., M. Z. Beckmann-Cavalcante, L. P. S. Brito, M. P. D. Lima e J. A. Osajima (2015) Conservação de inflorescências de helicônias previamente adubadas com doses crescentes de nitrogênio e potássio. Revista Caatinga 28:61-67, https://doi org/10.1590/1983-21252015v28n307ro

Arisha H. M. and A. Bardisi (1999) Effect of nitrogen fertilization and plant spacing on growth, yields and bud quality of common bean under sandy soil conditions. Zagazig Journal of Agricultural Research 26: 407-419.

Asrar A. A., G. M. Abdel-Fattah and K. M. Elhindi (2012) Improving growth, flower yield, and water relations of snapdragon (Antirhinum majus L.) plants grown under well-watered and water-stress conditions using arbuscular mycorrhizal fungi. Photosynthetica 50:305-316, https://doi.org/10.1007/s11099-012-0024-8

Baltazar B. 0., J. Zavala R. y S. J. Hernandez N. (2011) Producción Comercial de Heliconias. Colegio de Postgraduados. Montecillo, Texcoco, Edo. de México. 66 p.

Cardoso I. M. and T. W. Kuyper (2006) Mycorrhizas and tropical soil fertility. Agriculture, Ecosystems and Environment 116:72-84, https:// doi.org/10.1016/j.agee.2006.03.011

Clapperton M. J. and D. M. Reid (1992) A relationship between plant growth and increasing VA mycorrhizal inoculum density. New Phytologist 120:227-234, https://doi. org/10.1111/j.1469-8137.1992.tb05658.x

Cremlyn R. J. (1991) Agrochemicals: Preparation and Mode of Action. John Wiley and Sons. New York, USA. 396 p.

De Castro A. C. R., L. G. Walladino, V. Loges, M. F. A. de Castro and F. A. S. de Aragão (2015) Macronutrients deficiency in Heliconia psittacorum x Heliconia spathocircinata 'Golden Torch'. Revista Ciência Agronomica 46:258-265, https://doi. org/10.5935/1806-6690.20150005

De Farías A. P., A. W. de Albuquerque, G. Moura F. y L. S. Reis (2013) Produtividade da Heliconia psittacorum $x$ Heliconia pathocircinada cv. Golden Torch sob diferentes fontes de adubação orgânica. Revista Brasileira de Engenharia Agrícola e Ambiental 17:713-720, https://doi.org/10.1590/S141543662013000700004

El-Khateeb M. A., A. S. EL-Leithy and B. A. Aljemaa (2011) Effect of mycorrhizal fungi inoculation and humic acid on vegetative growth and chemical composition of Acacia saligna Labill. seedlings under different irrigation intervals. Journal of Horticultural Science and Ornamental Plants 3:283-289.

Gaur A., A. Gaur and A. Adholeya (2000) Growth and flowering in Petunia hybrida, Callistephus chinensis and Impatiens balsamina inoculated with mixed AM inocula or chemical fertilizers in a soil of low P fertility. Scientia Horticulturae 84:151-162, https:// doi.org/10.1016/S0304-4238(99)00105-3

Gaur A. and A. Adholeya (2005) Diverse response of five ornamental plant species to mixed indigenous and single isolate arbuscularmycorrhizal inocula in marginal soil amended with organic matter. Journal of Plant Nutrition 28:707-723, https://doi org/10.1081/PLN-200052647
Gerdemann J. W. and T. H. Nicolson (1963) Spores of mycorrhizal Endogone species extracted from soil by wet sieving and decanting. Transactions British Mycological Society 46:235244, https://doi.org/10.1016/S0007-1536(63)80079-0

Giovannetti M. and B. Mosse (1980) An evaluation of techniques for measuring vesicular arbuscular mycorrhizal infection in roots. New Phytologist 84:489-500, https://doi. org/10.1111/j.1469-8137.1980.tb04556.x

INEGI, Instituto Nacional de Estadística y Geografía (2015). Conjunto de datos vectoriales de la carta de uso del suelo y vegetación: escala 1: 250 000. https://www.inegi.org.mx/temas/ climatologia/ (Septiembre 2019).

INVAM, International Culture Collection of (Vesicular) Arbuscular Mycorrhizal Fungi (2016) Species descriptions from reference cultures. West Virginia University. Morgantown, West Virginia, USA. http:// fungi.invam.wvu.edu/the-fungi/species-descriptions.htm (September 2018)

Kuiper D. (1993) Sink strength: established and regulated by plant growth regulators. Plant Cell and Environment 16:1025-1026, https://doi.org/10.1111/j.1365-3040.1996.tb02052.x

Long L. K., Q. Yao, Y. H. Huang, R. H. Yang, J. Guo and H. H. Zhu (2010) Effects of arbuscular mycorrhizal fungi on zinnia and the different colonization between Gigaspora and Glomus. World Journal of Microbiology and Biotechnology 26:1527-1531, https://doi org/10.1007/s11274-010-0313-y

Marschner H. and B. Dell (1994) Nutrient uptake in mycorrhyzal symbiosis. Plant and Soil 159:89-102, https://doi.org/10.1007/ BF00000098

Malavolta E. (2006) Manual de Nutrição Mineral de Plantas. Editora UFV. São Paulo, Brasil. $631 \mathrm{p}$

Meir D., S. Pivonia, R. Levita, I. Dori, L. Ganot, S. Meir, ... H. Koltai (2010) Application of mycorrhizae to ornamental horticultural crops: lisianthus (Eustoma grandiflorum) as a test case. Spanish Journal of Agricultural Research 8:S5-S10, https://doi. org/10.5424/sjar/201008S1-1221

Montoya-Martínez A. C., G. Rincón-Enríquez, P. Lobit, L. López-Pérez and E. E. Quiñones-Aguilar (2019) Native arbuscular mycorrhizal fungi from the rhizosphere of Agave cupreata and their effect on Agave tequilana growth. Revista Fitotecnia Mexicana 42:429 438, https://doi.org/10.35196/rfm.2019.4.429-438

Mosse B. (1972) The influence of soil type and Endogone strain on the growth of mycorrhizal plants in phosphate-deficient soils. Review of the Ecological and Biological Society 9:529-537.

Mosse B. (1973) Advances in the study of vesicular-arbuscular mycorrhiza. Annual Review of Phytopathology 11:171-196, https://doi.org/10.1146/annurev.py.11.090173.001131

Nakano A., K. Takahashi and M. Kimura (2001) Effect of host shoot clipping on carbon and nitrogen sources for arbuscular mycorrhizal fungi. Mycorrhiza 10:287-293, https://doi.org/10.1007/ s005720000091

Perner H., D. Schwarz, C. Bruns, P. Mäder and E. George (2007) Effect of arbuscular mycorrhizal colonization and two levels of compost supply on nutrient uptake and flowering of pelargonium plants. Mycorrhiza 17:469-474, https://doi.org/10.1007/s00572-0070116-7

Phillips J. M. and D. S. Hayman (1970) Improved procedures for clearing roots and staining parasitic and vesicular-arbuscular mycorrhizal fungi for rapid assessment of infection. Transactions of the British Mycological Society 55:158-161, https://doi.org/10.1016/S0007-1536(70)80110-3

Poulton J. L., D. Bryla, R. T. Koide and A. G. Stephenson (2002) Mycorrhizal infection and high soil phosphorus improve vegetative growth and the female and male functions in tomato. New Phytologist 154: 255-264, https://doi.org/10.1046/j.1469 8137.2002.00366.x

Prasad K., A. Aggarwal, K. Yadav and A. Tanwar (2012) Impact of different levels of superphosphate using arbuscular mycorrhizal fungi and Pseudomonas fluorescens on Chrysanthemum indicum $\mathrm{L}$. Journal of Soil Science and Plant Nutrition 12:451-462, https:// doi.org/10.4067/S0718-95162012005000007

Rubí A. M., A. González H., A. M. Castillo G., V. Olalde P., B. G. Reyes R. y L. I. Aguilera G. (2009) Respuesta de Lilium sp. al fósforo y su relación con Glomus fasciculatum y Bacillus subtilis. Phyton 78:91-100 
Sahar, M. Zaghloul, E. M. Fatma, El-Quesni and A. A. M. Mazhar (2009) Influence of potassium humate on growth and chemical constituents of Thuja orientalis $L$ seedlings. Ozean Journal of Applied Sciences 2:73-78

SAS Institute (2011) SAS/STAT® 9.3 User's Guide. Statistical Analysis System Institute, Cary, North Carolina, USA. 8640 p.

Scagel C. F. (2003) Inoculation with arbuscular micorrhizal fungi alters nutrient allocation and flowering of Freesia $\times$ hybrida. Journal of Environmental Horticulture 21:196-205.

Scagel C. F. and R. P. Schreiner (2006) Phosphorus supply alters tuber composition, flower production, and mycorrhizal responsiveness of container-grown hybrid Zantedeschia. Plant and Soil 283:323-337, https://doi.org/10.1007/s11104-0060022-3

Schenck N. C. and Y. Pérez (1990) Manual for the Identification of VA Mycorrhizal Fungi. $3^{\text {rd }}$ edition. Synergistic Publications. Gainesville, Florida, USA. 286 p.

Sieverding E. (1983) Manual de Metodos para la Investigación de la Micorriza Vesículo-Arbuscular en el Laboratorio. Centro Internacional de Agricultura Tropical. Cali, Colombia. 121 p.

Sieverding E. and S. Toro (1985) The genus Entrophospora in Colombia. In: Physiological and Genetical Aspects of Mycorrhizae. V. Gianinazzi-Pearson and S. Gianinazzi (eds.). Proceedings of the 1st European Symposium on Mycorrhizae. Dijon, 1-5 July 1985. National Institute of Agricultural Research. Versailles, France. pp: 621-626

Smith S. F. and D. J. Read (2008) Mycorrhizal Symbiosis. 3rd edition. Academic Press. San Diego, California, USA. 800 p,

Sohn B. K., K. Y. Kim, S. J. Chung, W. S. Kim, S. M. Park, J. G. Kang, Y. S. Rim, J. S. Cho, T. H. Kim and J. H. Lee (2003) Effect of the different timing of AMF inoculation on plant growth and flower quality of chrysanthemum. Scientia Horticulturae 98:173-183, https:// doi.org/10.1016/S0304-4238(02)00210-8

Sosa R. T., J. Sánchez N., E. Morales G. and F. Cruz C. (2006) Interacción micorrizas arbusculares-Trichoderma harzianum (Moniliaceae) y efectos sobre el crecimiento de Brachiaria decumbens (Poaceae). Acta Biológica Colombiana 11:43-54.

Sosa R. F. M. (2013) Cultivo del género Heliconia. Cultivos Tropicales 34:24-32

Steiner A. A. (1973) The selective capacity of tomato plants for ions in a nutrient solution. Proceedings Third International Congress on Soilless Culture. Sassair, Italy. pp:43-54.

Tawaraya K., R. Hirose and T. Wagatsuma (2012) Inoculation of arbuscular mycorrhizal fungi can substantially reduce phosphate fertilizer application to Allium fistulosum L. and achieve marketable yield under field condition. Biology and Fertility of Soils 48:839-843, https://doi.org/10.1007/s00374-012-0669-2

Vaingankar J. D. and B. F. Rodrigues (2015) Effect of arbuscular mycorrhizal (AM) inoculation on growth and flowering in Crossandra infundibuliformis ( $L$.) Nees. Journal of Plant Nutrition 38:1478 1488, https://doi.org/10.1080/01904167.2014.957398

Van der Heijden M. G. A., J. N. Klironomos, M. Ursic, P. Moutoglis, R. StreitwolfEngel, T. Boller, A. Weimken and I. R. Sanders (1998) Mycorrhizal fungal diversity determines plant biodiversity, ecosystem variability and productivity. Nature 396:69-72, https://doi org/10.1038/23932

Varma A. and H. Schüepp (1994) Infectivity and effectiveness of Glomus intraradices on micropropagated plants. Mycorrhiza 5:29-37, https://doi.org/10.1007/BF00204017

Younis A., M. A. P. Khan and A. Riaz (2006) Effect of different levels of nitrogen, phosphorus, and potash fertilizers on growth of Dahlia coccinea cv. Decorative. Caderno de Pesquisa, Série Biologia 18:8-14.

Zhang X. H., A. J. Lin, Y. L. Gao, R. J. Reid, M. H. Wong and Y. G. Zhu (2009) Arbuscular mycorrhizal colonisation increases copper binding capacity of root cell walls of Oryza sativa L. and reduces copper uptake. Soil Biology and Biochemistry 41:930-935, https://doi org/10.1016/j.soilbio.2008.08.011 